\title{
Stochastic optimization of cost-risk for integrated energy system considering wind and solar power correlated
}

\author{
Jiehui ZHENG ${ }^{1}$, Yanni KOU ${ }^{1}$, Mengshi LI $^{1}$, Qinghua WU ${ }^{1}$
}

\begin{abstract}
Due to the growing penetration of renewable energies (REs) in integrated energy system (IES), it is imperative to assess and reduce the negative impacts caused by the uncertain REs. In this paper, an unscented transformation-based mean-standard (UT-MS) deviation model is proposed for the stochastic optimization of cost-risk for IES operation considering wind and solar power correlated. The unscented transformation (UT) sampling method is adopted to characterize the uncertainties of wind and solar power considering the correlated relationship between them. Based on the UT, a mean-Standard (MS) deviation model is formulated to depict the trade-off between the cost and risk of stochastic optimization for the IES optimal operation problem. Then the UT-MS model is tackled by a multi-objective group search optimizer with adaptive covariance and Lévy flights embedded with a multiple constraints handling technique (MGSO-ACL-CHT) to ensure the feasibility of Peratooptimal solutions. Furthermore, a decision making method,
\end{abstract}

CrossCheck date: 25 January 2019

Received: 26 May 2018/Accepted: 25 January 2019/Published online: 20 July 2019

(C) The Author(s) 2019

Mengshi LI

mengshili@scut.edu.cn

Jiehui ZHENG

zhengjh@scut.edu.cn

Yanni KOU

epyn.kou@mail.scut.edu.cn

Qinghua WU

wuqh@scut.edu.cn

1 School of Electric Power Engineering, South China University of Technology, Guangzhou 510640, China improve entropy weight (IEW), is developed to select a final operation point from the set of Perato-optimal solutions. In order to verify the feasibility and efficiency of the proposed UT-MS model in dealing with the uncertainties of correlative wind and solar power, simulation studies are conducted on a test IES. Simulation results show that the UT-MS model is capable of handling the uncertainties of correlative wind and solar power within much less samples and less computational burden. Moreover, the MGSOACL-CHT and IEW are also demonstrated to be effective in solving the multi-objective UT-MS model of the IES optimal operation problem.

Keywords Integrated energy system, Renewable energies, Unscented transformation, Mean-standard deviation, Multi-objective optimization, Decision making

\section{Introduction}

In recent years, due to the fast depletion and severe pollution of fossil fuels, there is a massive stimulation to integrate the renewable energies (REs) such as wind and solar into integrated energy system (IES) [1]. However, the growing penetration of wind and solar power imposes challenges to the reliability and efficiency of IES operation since these resources are neither schedulable nor fully predictable $[2,3]$. In this regard, it is necessary to consider the uncertainties aroused by REs and to minimize the stochastic impact in the IES optimal operation problem [4].

In the aspect of accommodating the uncertainties of REs in the IES operation, there are two main kinds of stochastic approaches, namely, the fuzzy approach and the probabilistic approach [5]. Among the fuzzy optimization approaches [6-8], the wind and solar power are depicted in 
fuzzy set notations using membership functions [9]. Although the fuzzy approach is easy to implement, it might be subjected to the dispatchers' attitude and trapped in selecting suitable membership functions $[10,11]$. In terms of the probabilistic approach, it can be generally divided into scenario-based programming [12-16], robust optimization $[17,18]$, chance-constrained optimization $[19,20]$ and risk-based optimization [21,22].

Among these approaches, the scenario-based stochastic optimization method is quite cost-effective. It considers the system uncertainties as random input variables and represents their probability distributions by scenarios generated by Monte Carlo sampling (MCS) [23] or Latin hypercube sampling (LHS) [24]. Each scenario is characterized as a deterministic problem that can be solved by mathematical programming methods or evolutionary optimization algorithms [25]. However, the computation burden of the original problem increases along with the number of scenarios. Although the scenario reduction (SR) method [26] that aggregates similar scenarios can reduce the computation burden, it also reduces the accuracy in calculating the actual operating cost.

To relieve the computational burden of scenario-based stochastic optimization, it is necessary to select the representative scenarios that can fully reflect the properties of input variables' probabilistic uncertainties. The unscented transformation (UT) sampling method, which is raised for nonlinear transformation in filters and estimators [27], is adopted to characterize the uncertainties of wind and solar power considering the correlated relationship between them [28]. The UT is a reliable method for calculating the statistic information of output variables through a set of nonlinear transformations. This method handles nonlinear systems well since no linearization process is needed. By constructing a set of sample points known as sigma points to exhibit specific properties of input variables, the UT is able to obtain the means and variances of output variables with limited numbers of calculation by treating the sample points as representative scenarios.

Based on the UT, this paper attempts to formulate the mean-standard deviation (MS) model to depict trade-off between the cost and risk of stochastic optimization for the microgrid optimal operation problem. The MS model is similar to the mean-variance model proposed by Markowitz for portfolio optimization problem which considers both the cost and risk in the uncertain environment [29]. However, the UT-MS model requires less sample points compared with the mean-variance model, which will reduce numbers of repetitive computation and relieve the computational burden for the IES optimal operation problem.

Accordingly, the IES optimal operation problem is mathematically formulated as a multi-objective optimization problem. Moreover, the problem also contains a variety of constraints to satisfy the operation requirements of IES. Then, an algorithm named multi-objective group search optimizer with adaptive covariance and Lévy flights (MGSO-ACL) [30] is employed to find the Paretooptimal solutions. In addition, a multiple constraints handling technique is embedded to the MGSO-ACL in this paper (MGSO-ACL-CHT) to overcome the deficiency of MGSO-ACL in dealing with multiple constraints. The constraint handling method regards the overall constraint violation degree as an objective and modifies the Pareto dominated rule accordingly. To the end, a decision making method named improved entropy weight (IEW) is applied to determine the final operation point from the set of Pareto-optimal solutions.

The rest of this paper is organized as follows: Section 2 formulates the uncertainty characterization of wind and solar power using the UT-MS model for the IES optimal operation problem. Section 3 presents the multi-objective optimization algorithm MGSO-ACL-CHT to optimization the UT-MS model, and develops the decision making method named IEW to select a final operation point for he IES optimal operation problem. Simulation studies are conducted in Section 4 to verify the effectiveness of the proposed method. Finally, the conclusions are drawn in Section 5 .

\section{Uncertainty characterization using UT-MS model}

Due to the stochastic nature of wind and solar power, it is required to model these variables within a probabilistic environment in the optimal operation of IES. And the system operator should be able to acquire the statistic information of output variables, such as the operation cost and state variables, through the statistic information of the input random variables, such as wind and solar power.

The IES optimal operation problem studied in this paper conducts in one-hour interval, and the power generated by the wind turbine (WT) and photovoltaic (PV) generators are represented by their day-ahead forecast results. Reference [31] applied the Gaussian distribution to describe the wind forecast error, then combined the probability density function (PDF) of forecast error with the forecast value to obtain the distribution of actual wind power output. However, the variable range of Gaussian distribution is infinite while the power output of WT generator has its limits. As a consequence, the Gaussian distribution might be unsuitable to represent the wind forecast error. It has been demonstrated that the Beta distribution owes advantages over the Gaussian distribution in approximating the forecast error of wind power in [32], therefore, we adopt the 
Beta distribution to model the forecast errors of wind power and the corresponding PDF is given by the following equations:

$f\left(P_{\mathrm{w}_{i}}\right)=\frac{\Gamma\left(\alpha_{i}+\beta_{i}\right)}{\Gamma\left(\alpha_{i}\right) \Gamma\left(\beta_{i}\right)}\left(\frac{P_{\mathrm{w}_{i}}}{P_{\mathrm{w}_{i}}^{\max }}\right)^{\alpha_{i}-1}\left(1-\frac{P_{\mathrm{w}_{i}}}{P_{\mathrm{w}_{i}}}\right)^{\beta_{i}-1}$

where $P_{\mathrm{w}_{i}}$ and $P_{\mathrm{w}_{i}}^{\max }$ are the forecast and maximum wind power output at the $i^{\text {th }}$ bus; $\Gamma(\cdot)$ is the Gamma function, $\alpha_{i}$ and $\beta_{i}$ are the shape parameters of the PDF at the $i^{\text {th }}$ bus which are related to the wind power forecast parameters $\sigma_{\mathrm{w}_{i}}^{2}$ (variance) and $\mu_{\mathrm{w}_{i}}$ (mean) [32]. The following equations show these relationships:

$\alpha_{i}=\frac{\mu_{\mathrm{w}_{i}}^{2}\left(1-\mu_{\mathrm{w}_{i}}\right)}{\sigma_{\mathrm{w}_{i}}^{2}}-\mu_{\mathrm{w}_{i}}$

$\beta_{i}=\alpha_{i} \frac{1-\mu_{\mathrm{w}_{i}}}{\mu_{\mathrm{w}_{i}}}$

In this paper, the forecast values of solar radiation taking into account the forecast error is formulated by Gaussian distribution [33]:

$f\left(r_{i}\right)=\frac{1}{\sqrt{2 \pi \sigma_{\mathrm{r}_{i}}^{2}}} \exp \left(-\frac{\left(r_{i}-\mu_{\mathrm{r}_{i}}\right)^{2}}{2 \sigma_{\mathrm{r}_{i}}^{2}}\right)$

where $r_{i}$ is the forecast solar radiation $\left(\mathrm{W} / \mathrm{m}^{2}\right)$ at the $i^{\text {th }}$ bus; $\sigma_{\mathrm{r}_{i}}^{2}$ and $\mu_{\mathrm{r}_{i}}$ are the variance and mean of the solar radiation forecast result at the $i^{\text {th }}$ bus, respectively.

A relationship between the power generated and solar radiation was presented in [34], which is calculated as:

$P_{\mathrm{s}_{i}}\left(r_{i}\right)=A_{i} r_{i} \eta$

where $P_{\mathrm{s}_{i}}$ is the power output of PV generators at the $i^{\text {th }}$ bus; $A_{i}$ is the total size of the solar panels in the solar farm at the $i^{\text {th }}$ bus; $\eta$ is the convergence rate of the solar panel which is dependent on the incident radiation and the measured ambient temperature, and it is simply set to be 0.14 in this paper.

As the PV generator power output function is a linear function, the PDF of solar power forecast values is also described by Gaussian distribution:

$f\left(P_{\mathrm{s}_{i}}\right)=\frac{1}{\sqrt{2 \pi \sigma_{\mathrm{s}_{i}}^{2}}} \exp \left(-\frac{\left(P_{\mathrm{s}_{i}}-\mu_{\mathrm{s}_{i}}\right)^{2}}{2 \sigma_{\mathrm{s}_{i}}^{2}}\right)$

where $\sigma_{\mathrm{s}_{i}}^{2}=\left(\eta A_{i} \sigma_{\mathrm{r}_{i}}\right)^{2}$ and $\mu_{\mathrm{s}_{i}}=\eta A_{i} \mu_{\mathrm{r}_{i}}$ are the variance and mean of the solar power forecast result at the $i^{\text {th }}$ bus, respectively.

Suppose there are $p$ wind farms and $q$ solar farms in a regional IES, then the input variables $\boldsymbol{z}=\left[z_{1}, z_{2}, \ldots, z_{n}\right]$ $=\left[P_{\mathrm{w}_{1}}, P_{\mathrm{w}_{2}}, \ldots, P_{\mathrm{w}_{p}}, P_{\mathrm{s}_{1}}, P_{\mathrm{s}_{2}}, \ldots, P_{\mathrm{s}_{q}}\right]$, where $n=p+q$. According to the PDFs of wind and solar power forecasts, the mean value of $\boldsymbol{z}$ is denoted as $\overline{\boldsymbol{z}}=\boldsymbol{\mu}=$ $\left[\mu_{\mathrm{w}_{1}}, \mu_{\mathrm{w}_{2}}, \ldots, \mu_{\mathrm{w}_{p}}, \mu_{\mathrm{s}_{1}}, \mu_{\mathrm{s}_{2}}, \ldots, \mu_{\mathrm{s}_{q}}\right]$, and the covariance of $z$ is established as:

$\boldsymbol{C}_{\mathrm{zz}}=\left[\begin{array}{cccc}\sigma_{1}^{2} & \phi_{12} \sigma_{1} \sigma_{2} & \cdots & \phi_{1 n} \sigma_{1} \sigma_{n} \\ \phi_{21} \sigma_{2} \sigma_{1} & \sigma_{2}^{2} & \cdots & \phi_{2 n} \sigma_{2} \sigma_{n} \\ \vdots & \vdots & & \vdots \\ \phi_{n 1} \sigma_{n} \sigma_{1} & \phi_{n 2} \sigma_{n} \sigma_{2} & \cdots & \sigma_{n}^{2}\end{array}\right]$

where $\sigma_{i}$ is the standard deviation (STD) of the $i^{\text {th }}$ input variable; $\phi_{i j}$ is the correlation coefficient between the $i^{\text {th }}$ and $j^{\text {th }}$ input variable, that is,

$\phi_{i j}=\frac{\operatorname{cov}\left(z_{i}, z_{j}\right)}{\sigma_{i} \sigma_{j}}=\frac{E\left[\left(z_{i}-\mu_{i}\right)\left(z_{j}-\mu_{j}\right)\right]}{\sigma_{i} \sigma_{j}}$

The correlation coefficient is in the range of $[-1,1]$, indicating the degree of linear dependence between two variables. The correlation of wind and solar power is reasonable since they are all influenced by the weather conditions. It is shown that wind and solar power are negatively correlated on all time scales, and the hourly correlation coefficient of wind and solar power is evaluated to be -0.2 [35]. This suggests that more windy time are less sunny, and vice versa. Note that the assumption on the correlation of wind and solar power is based on the geographical location closely enough under the similar weather pattern. Accordingly, the correlation coefficient in (5) is suitable for the distributed network in a regional IES.

With the statistic information $\bar{z}$ and $\boldsymbol{C}_{\mathrm{zz}}$ of input variables, the UT method employs three steps to establish the MS dispatch model, that is, sampling, nonlinear

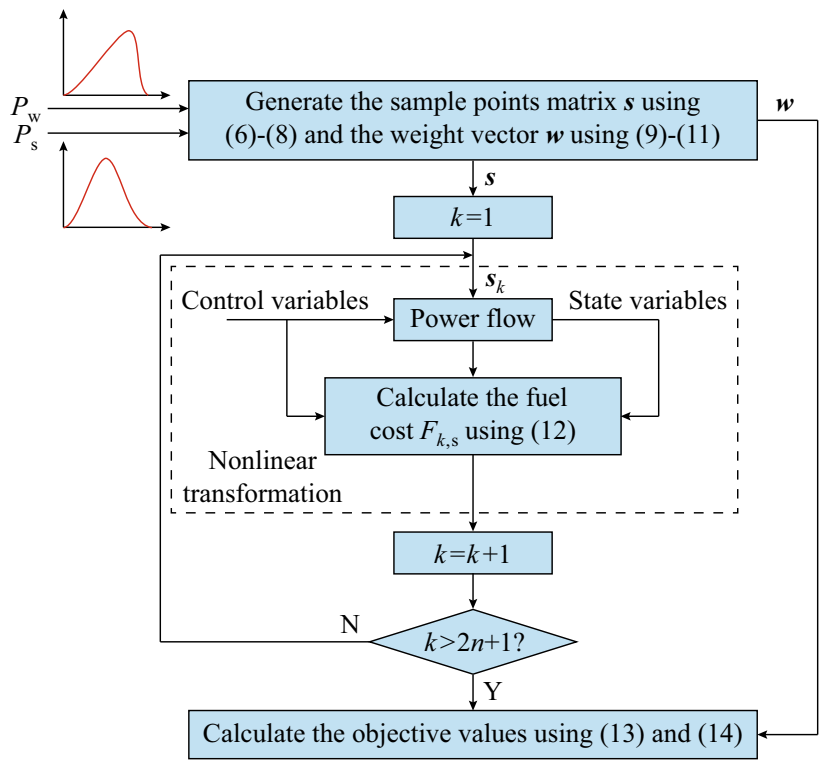

Fig. 1 Flowchart of UT method for calculating objective values of MS model 
transformation, and outputting objective values, as illustrated in Fig. 1. The first step is to generate the sample points matrix $\boldsymbol{s}$ and the corresponding weight vector $\boldsymbol{w}$. The $2 n+1$ samples of input variables are obtained as:

$\boldsymbol{s}_{k}=\boldsymbol{\mu}+\sqrt{\frac{n}{1-w_{0}}} \boldsymbol{c h o l}(k,:) \quad k=1,2, \ldots, n$

$\boldsymbol{s}_{k+n}=\boldsymbol{\mu}-\sqrt{\frac{n}{1-w_{0}}} \operatorname{chol}(k,:) \quad k=1,2, \ldots, n$

$\boldsymbol{s}_{2 n+1}=\boldsymbol{\mu}$

where $\boldsymbol{s}_{k}$ is the $n$-dimensional $k^{\text {th }}$ sample of input variables; $w_{0}$ is the weight of central moments sample; and chol $=$ $\sqrt{\boldsymbol{C}_{\mathrm{zz}}}$ is the square-root matrix with its $k^{\text {th }}$ row presented by $\operatorname{chol}(k,:)$.

The weight corresponding to each sample is calculated as follows:

$$
\begin{aligned}
& w_{k}=\frac{1-w_{0}}{2 n} \quad k=1,2, \ldots, n \\
& w_{k+n}=\frac{1-w_{0}}{2 n} \quad k=1,2, \ldots, n \\
& w_{2 n+1}=w_{0}
\end{aligned}
$$

The second step is nonlinear transformation to yield a set of transformed output sample points, as shown in the dotted box of Fig. 1. The control variables which are scenario-independent, and each sample point $\boldsymbol{s}_{k}$ which contains sampling values of wind and solar power, are input into the power flow. After running the NewtonRaphson power flow program, the sampling values of state variables can be determined. In this paper, it is assumed that the wind and solar farms are owned by the grid company, then the objective function $F$ that the regional IES needs to minimize is the total fuel cost of thermal generators:

$F=\sum_{i=1}^{N_{\mathrm{G}}}\left(a_{i} P_{\mathrm{G}_{i}}^{2}+b_{i} P_{\mathrm{G}_{i}}+c_{i}\right)$

where $P_{\mathrm{G}_{i}}$ is the real power output of $i^{\text {th }}$ generator; $a_{i}, b_{i}$ and $c_{i}$ are the cost coefficients of $i^{\text {th }}$ generator; $N_{\mathrm{G}}$ is the number of generators.

Therefore, with the control variables and the obtained state variables, $F_{k}$, the total fuel cost $F$ of the $k^{\text {th }}$ sample $\boldsymbol{s}_{k}$, can be calculated. Although the costs of wind and solar power are not taken into consideration, the uncertain wind and solar power will result in the uncertainty of the entire system, hence $F$ varies in different samples. After the calculation of the $2 n+1$ sample values of $F$, the mean value and STD of $F$ corresponding to $2 n+1$ sample points,
$\bar{F}, \sigma_{F}$, can be obtained by summing the weighted sample values:

$$
\begin{aligned}
& \bar{F}=\sum_{k=1}^{2 n+1} w_{k} F_{k} \\
& \sigma_{F}=\left[\sum_{k=1}^{2 n+1} w_{k}\left(F_{k}-\bar{F}\right)^{2}\right]^{\frac{1}{2}}
\end{aligned}
$$

From the above procedure, it is clear that the UT method is very easy to implement and computationally efficient since it only needs $2 n+1$ sample points to characterize $n$ uncertain variables, while MCS and LHS always needs hundreds of sample points. Furthermore, the UT owns the additional advantage in tackling the correlation of random variables. To accomplish the correlation treatment task, the UT method only need to conduct Choeskely decomposition on $\boldsymbol{C}_{\mathrm{zz}}$ and substitute $\sqrt{\boldsymbol{C}_{\mathrm{zz}}}$ into (6) and (7), avoiding the complicated processes such as orthogonal transformation which is necessary for MCS and LHS.

In order to minimize the operation cost which guaranteeing the optimal solution is stable and suitable under the fluctuation of wind and solar power, the deviation of operation cost is simultaneously set as the objective function. Hence the model is formulated as a multi-objective cost-risk optimization problem. The two indices in (13) and (14) quantify the cost and risk of a system, respectively, then they constitute the objectives of the MS model which is formulated as:

$\min \left[\begin{array}{ll}\bar{F} & \sigma_{F}\end{array}\right]$

s.t.

$\left\{\begin{array}{c}\boldsymbol{g}\left(\boldsymbol{u}, \boldsymbol{s}_{k}, \boldsymbol{x}_{k}\right)=\mathbf{0} \\ \boldsymbol{h}_{1} \leq \boldsymbol{h}\left(\boldsymbol{u}, \boldsymbol{x}_{k}\right) \leq \boldsymbol{h}_{\mathrm{u}}\end{array} \quad k=0,1, \ldots, 2 n\right.$

In (16), the vector $\boldsymbol{u}$ consists of control variables which include the real power of each generator $P_{\mathrm{G}}$ (excluding the power of slack bus $P_{\mathrm{G}_{1}}$ ), the voltages of generator bus $V_{\mathrm{G}}$, the tap ratios of transformers $T_{m}$ and the reactive power generation of voltampere reactive (var) sources $Q_{\mathrm{C}}$. The vector $\boldsymbol{x}_{k}$ consists of state variables in the $k^{\text {th }}$ sample $\boldsymbol{s}_{k}$, and the state variables include the slack bus power $P_{\mathrm{G}_{1}}$, the voltage of load bus $V_{\mathrm{L}}$, the generator reactive power $Q_{\mathrm{G}}$ and the apparent power flow $S_{\mathrm{L}}$. It should be noted that the uncertainty of input variables causes all the state variables to be uncertainty, hence these constraints must be satisfied in all scenarios represented by sigma points.

The equality constraints $\boldsymbol{g}$ are the balance limits of active and reactive power described by a set of non-linear power flow equations between bus $i$ and $j$, and they can be satisfied during the running of Newton-Raphson power flow program [34]. 


$$
\begin{aligned}
& P_{\mathrm{G}_{i}}-P_{\mathrm{D}_{i}}-V_{i} \sum_{j \in N_{i}}\left(G_{i j} \cos \theta_{i j}+B_{i j} \sin \theta_{i j}\right)=0 \\
& Q_{\mathrm{G}_{i}}-Q_{\mathrm{D}_{i}}-V_{i} \sum_{j \in N_{i}}\left(G_{i j} \sin \theta_{i j}-B_{i j} \cos \theta_{i j}\right)=0
\end{aligned}
$$

The inequality constraints $\boldsymbol{h}$ are associated with the distributed generators, transformers, shunt compensators and transmission lines, respectively. The corresponding limits of active power, reactive power and voltage of generators $\mathrm{G}_{i}, i=1,2, \ldots, N_{\mathrm{G}}$, are given by:

$P_{\mathrm{G}_{i}}^{\min } \leq P_{\mathrm{G}_{i}} \leq P_{\mathrm{G}_{i}}^{\max }$

$Q_{\mathrm{G}_{i}}^{\min } \leq Q_{\mathrm{G}_{i}} \leq Q_{\mathrm{G}_{i}}^{\max }$

$V_{\mathrm{G}_{i}}^{\min } \leq V_{\mathrm{G}_{i}} \leq V_{\mathrm{G}_{i}}^{\max }$

In addition, the constraints of transformers, shunt compensators and transmission lines are given as follows:

$$
\begin{array}{ll}
T_{m}^{\min } \leq T_{m} \leq T_{m}^{\max } & m=1,2, \ldots, N_{\mathrm{T}} \\
Q_{\mathrm{C}_{n}}^{\min } \leq Q_{\mathrm{C}_{n}} \leq Q_{\mathrm{C}_{n}}^{\max } & n=1,2, \ldots, N_{\mathrm{C}} \\
V_{\mathrm{L}_{u}}^{\min } \leq V_{\mathrm{L}_{u}} \leq V_{\mathrm{L}_{u}}^{\max } & u=1,2, \ldots, N_{\mathrm{L}} \\
\left|S_{\mathrm{L}_{v}}\right| \leq S_{\mathrm{L}_{v}}^{\max } & v=1,2, \ldots, N_{1}
\end{array}
$$

where $N_{\mathrm{T}}, N_{\mathrm{C}}, N_{\mathrm{L}}$ and $N_{1}$ are the number of transformers, shunt compensators, load buses and transmission lines, respectively.

It should be noted that the control variables which are scenario-independent can be initialized within their boundary limits, while the uncertainty of input variables causes all the state variables to be uncertainty, hence these constraints must be satisfied in all scenarios represented by sigma points. The detailed constraint handling method for the state variables will be introduced in the following section.

\section{Methodology and implementation}

With the related work done in Section 2, the MGSOACL-CHT optimization algorithm for optimizing the UTMS model and the IEW decision making method for selecting the best compromise solution of Pareto-optimal front are described in this section.

\subsection{Multi-objective optimization}

The proposed UT-MS model is a multi-objective optimization problem which has a set of non-dominated, alternative solutions, known as the Pareto-optimal set, instead of a single global optimal solution. The traditional mathematical method is a serial algorithm characterized with single point search, thus cannot utilize the concept of Pareto-optimality to evaluate solutions [36]. Multi-objective evolutionary algorithms proposed in recent decades, such as the non-dominated sorting genetic algorithm-II (NSGA-II) [37], multi-objective particle swarm optimizer [38], multi-objective differential evolution algorithm, MGSO-ACL [30] can search for multiple solutions in parallel and are insensitive to the shape of the objective functions such as discontinuity, non-convexity, multiple modality, non-uniformity of the search space [39], and they have been successfully applied in power system. Considering the good performance of MGSO-ACL, it is utilized by this paper to optimize the proposed model. The detailed description of its mechanism can refer to the previous work in [30].

A common feature of evolutionary algorithms is that it is convenient for them to tackle the boundary limits of individuals composed by control variables, however, as to the constraints given by the state variables, the penalty method is frequently employed [40]. Nonetheless, the penalty is always added to one of the many objectives of MOP and the penalty factor terms are required to be set sufficiently large, which might hinder the evolutionary process of the objective being punished. What's more, in the stochastic multi-objective optimization problem, the valid trail solution must be feasible for all constraints in all scenarios, which is difficulty for the penalty method to satisfy. Therefore, a multiple constraints handling technique is proposed to tackle the boundary limits of state variables.

In this paper, the state variables are real power generation output at slack bus $P_{\mathrm{G}_{1}}$, load bus voltage magnitude $V_{\mathrm{L}}$, reactive power generation output of thermal units $Q_{\mathrm{G}}$ and transmission line loading $S_{\mathrm{L}}$, then the constraint violation degree $f^{\text {viol }}$ is expressed as:

$f^{\mathrm{viol}}=\lambda_{\mathrm{P}} P_{\mathrm{G}_{1}}^{\mathrm{lim}}+\lambda_{\mathrm{V}} \sum_{u=1}^{N_{\mathrm{L}}} V_{\mathrm{L}_{u}}^{\mathrm{lim}}+\lambda_{\mathrm{Q}} \sum_{i=1}^{N_{\mathrm{G}}} Q_{\mathrm{G}_{i}}^{\mathrm{lim}}+\lambda_{\mathrm{S}} \sum_{v=1}^{N_{\mathrm{l}}} S_{\mathrm{L}_{v}}^{\lim }$

where $\lambda_{\mathrm{P}}, \lambda_{\mathrm{V}}, \lambda_{\mathrm{Q}}$ and $\lambda_{\mathrm{S}}$ are the weights used to convert the various violations into basically the same magnitude, and they are different from the penalty factor terms in [40] which should be set sufficiently large to achieve the effect of punishment; $x^{\lim }$ is the violation of state variable $x$,

$x^{\lim }=\left\{\begin{array}{cc}x-x^{\max } & x>x^{\max } \\ x^{\min }-x & x<x^{\min } \\ 0 & x^{\min } \leq x \leq x^{\max }\end{array}\right.$

where $x^{\min }$ and $x^{\max }$ are the lower and upper limits of state variable $x$, respectively. 
Under the uncertain environment, these constraints must be satisfied in all scenarios represented by sigma points, then the mean constraint violation $\bar{f}^{\text {viol }}$ shown in (28) must be equal to 0 when the optimization process terminates.

$\bar{f}^{\mathrm{viol}}=\frac{1}{2 n+1} \sum_{k=1}^{2 n+1} f_{k}^{\mathrm{viol}}$

where $f_{k}^{\text {viol }}$ is the constraint violation $f^{\text {viol }}$ in the $k^{\text {th }}$ sample $\boldsymbol{s}_{k}$, which can be calculated during the nonlinear transformation process.

Then $\bar{f}^{\text {viol }}$ is regarded as an independent objective in the optimization process, and this constraint handling method can guide the individuals to evolve towards the feasible regions more effectively compared with penalty method.

The original Pareto dominated rule should be modified accordingly to promote the priority of constraints. The constrained Pareto-dominance principle [41] is described as follows: The feasible individual always dominates the infeasible one, the individual with small violation always dominates the one with big violation. For two individuals $X_{1}$ and $X_{2}$, there is $X_{1} \succ X_{2}$ if and only if one of the following conditions is satisfied, (1) $f^{\mathrm{viol}}\left(X_{1}\right)=0, f^{\mathrm{viol}}\left(X_{2}\right)$ $>0$; (2) $f^{\mathrm{viol}}\left(X_{1}\right)>f^{\mathrm{viol}}\left(X_{2}\right)>0$; (3) $f^{\mathrm{viol}}\left(X_{1}\right)=f^{\mathrm{viol}}\left(X_{2}\right)$ $=0$ and for each objective $i, f_{i}\left(X_{1}\right)<f_{i}\left(X_{2}\right)$.

The stochastic dispatch is optimized based on certain number of objective function evaluations. Before the evaluation, the UT is utilized to generate $2 n+1$ sample points of the wind and solar power. Trial solutions are evaluated under these scenarios to calculate the fuel cost and constraints violation. The detailed procedure is outlined in Algorithm 1. And the Pseudo code of MGSOACL-CHT algorithm with constrained Pareto-dominance

Algorithm 1 Pseudo code of fitness evaluation

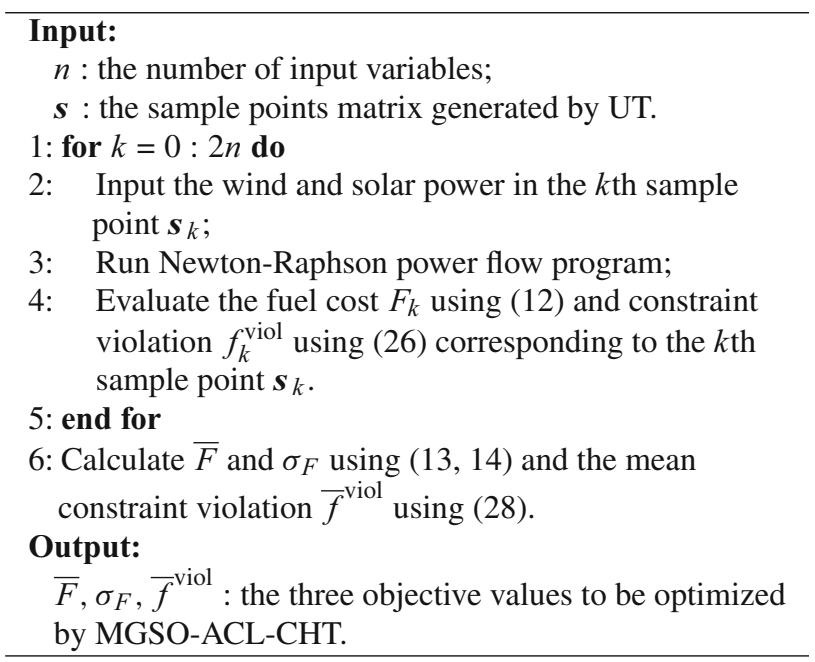

Algorithm 2 Pseudo code of MGSO-ACL-CHT for the UT-MS model

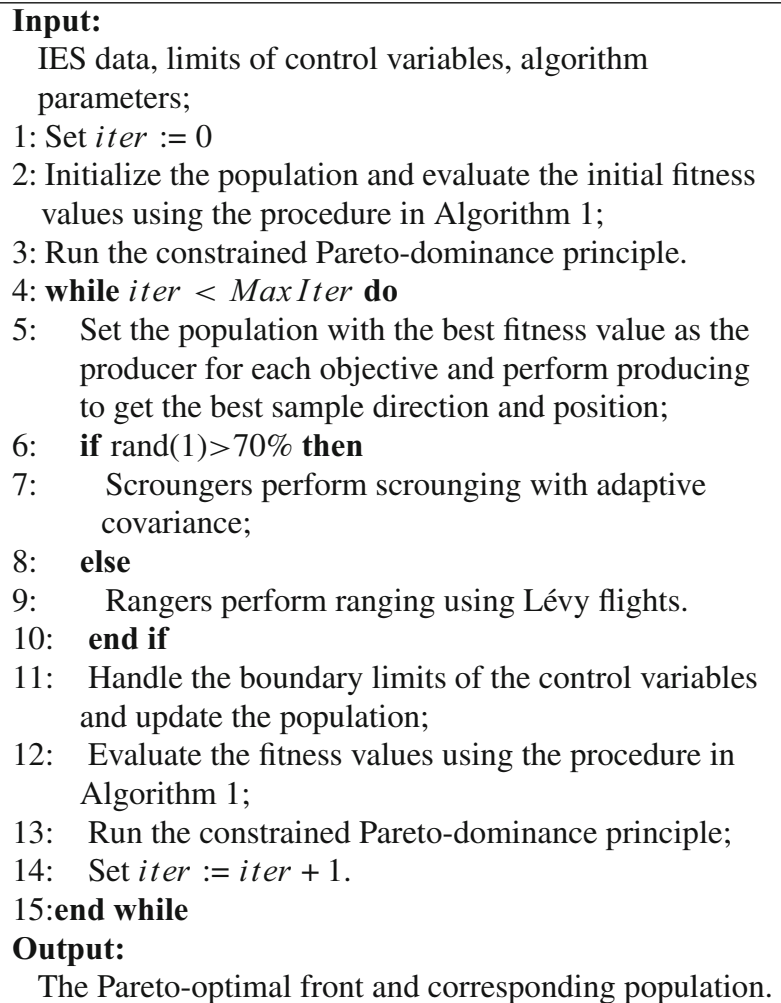

principle for tackling the UT-MS model is displayed in Algorithm 2.

\subsection{IEW method and its decision making model}

Entropy is originally the concept in thermodynamics, and it is C. E. Shannon that introduced this concept into the area of information theory [42]. Utilizing the characteristic of entropy that it can measure the quantity of the useful information provided by the data, the entropy weight (EW) method can be used to assign objective weights. However, the EW method would endow the wrong weights when all the entropy values of attributes are close to 1 . To overcome the disadvantages of the original EW method, we propose an IEW method and apply it to assess the solutions with multiple objective (attribute) values. The detailed illustration of IEW method is given as follows:

1) Denote the Pareto-optimal set obtained by multi-objective optimization algorithms as $X_{n m}$, it contains $n$ solutions represented by rows and $m$ objectives represented by columns. Normalize $x_{n m}$ into $r_{n m}$ as below.

For minimization problem:

$$
r_{i j}=\left(\max _{1 \leq i \leq n} x_{i j}-x_{i j}\right) /\left(\max _{1 \leq i \leq n} x_{i j}-\min _{1 \leq i \leq n} x_{i j}\right)
$$


For maximization problem:

$r_{i j}=\left(x_{i j}-\min _{1 \leq i \leq n} x_{i j}\right) /\left(\max _{1 \leq i \leq n} x_{i j}-\min _{1 \leq i \leq n} x_{i j}\right)$

where $r_{i j}$ denotes the normalized value of the $j^{\text {th }}$ objective in the $i^{\text {th }}$ solution.

2) Calculate the entropy value for each objective using (31).

$$
\left\{\begin{array}{c}
H_{j}=-k \sum_{i=1}^{n} f_{i j} \ln f_{i j} \\
f_{i j}=r_{i j} / \sum_{i=1}^{n} r_{i j}
\end{array}\right.
$$

where $0 \leq H_{j} \leq 1$; and it is assumed that if $\ln f_{i j}=0$, $f_{i j} \ln f_{i j}=0 ; i=1,2, \cdots, n ; j=1,2, \cdots, m$.

3) Employ (32) and (33) to calculate $\omega_{j}$ that should be assigned to objective $j$.

$$
\begin{aligned}
& \left\{\begin{array}{c}
\omega_{j, \mathrm{e}}=\left\{\begin{array}{cc}
(1-\bar{H}) \omega_{j, \mathrm{e} 1}+\bar{H} \omega_{j, \mathrm{e} 2} & H_{j}<1 \\
0 & H_{j}=1
\end{array}\right. \\
\omega_{j, \mathrm{e} 1}=\left(1-H_{j}\right) / \sum_{j=1, H_{j} \neq 1}^{m}\left(1-H_{j}\right)
\end{array}\right. \\
& \omega_{j, \mathrm{e} 2}=\left(1 / H_{j}\right) / \sum_{j=1, H_{j} \neq 0}^{m}\left(1 / H_{j}\right) \\
& \omega_{j}=\omega_{j, \mathrm{~s}} \omega_{j, \mathrm{e}} / \sum_{j=1}^{m} \omega_{j, \mathrm{~s}} \omega_{j, \mathrm{e}}
\end{aligned}
$$

where $\bar{H}$ is the mean value of all the entropy values which are not equal to $1 ; 0 \leq \omega_{j, \mathrm{e}} \leq 1 ; \sum_{j=1}^{m} \omega_{j, \mathrm{e}}=1 ; \omega_{j, \mathrm{~s}}$ is the subjective weight of attribute $j$.

4) Calculate the aggregation assessment value $u_{i}$ for each solution using below equation.

$u_{i}=\sum_{j=1}^{m} \omega_{j} r_{i j} \quad i=1,2, \ldots, n$

5) Rank solutions according to the aggregation assessment values, and a larger value indicates a better solution.

\section{Simulation studies}

\subsection{System description and parameter settings}

In this section, a test regional IES, which consists of a modified IEEE 30-bus system embedded with WT and PV generators [34], is utilized to verify the effectiveness of the UT method in tackling uncertainty, and the performance of MGSO-ACL algorithm with constrained Pareto-dominance principle in solving MS model. The wind farms are
Table 1 Parameters of WT and PV generators

\begin{tabular}{lcll}
\hline Generator & Node & $\mu_{\mathrm{w}}(\mathrm{MW})$ & $\sigma_{\mathrm{s}}(\mathrm{MW})$ \\
\hline WT generators & 5 & 12.7 & 1.27 \\
& 11 & 30 & 3.0 \\
& 13 & 20.9 & 2.09 \\
PV generators & 2 & 12.5 & 2.50 \\
& 17 & 6.7 & 1.34 \\
& 23 & 8.4 & 1.68 \\
\hline
\end{tabular}

installed on buses 5, 11 and 13, and the original thermal units on these buses are shut down for energy saving; the PV generators are located in buses 1, 17 and 23. The forecasts of wind and solar power are assumed to be available, the mean $\mu_{\mathrm{w}}$ and STD $\sigma_{\mathrm{w}}$ of wind forecast result, and the mean $\mu_{\mathrm{s}}$ and STD $\mu_{\mathrm{s}}$ of solar forecast result are outlined in Table 1. As for MGSO-ACL algorithm, it is evaluated in comparisons with the NSGA-II, their population sizes are all set to be 50 and the maximum number of iterations 200. All the simulation studies are implemented on a personal computer with a $3.5 \mathrm{GHz}$ processor and a 4 GB of RAM using MATLAB.

\subsection{Case 1: The uncorrelated input variables}

In this case, it is assumed that the wind and solar power are independent with each other, that is, the correlation coefficient $\phi_{i j}$ in (5) is equal to 0 for $i \neq j$. As the LHS method has been proved effective in handling uncertainty [29, 43], it is utilized here as a criterion to test the accuracy of the UT method and the widely used SR method [26]. We set the sample number of LHS method to be 300 , and the SR method to be 13 which is equal to the sample number of the UT method. The Pareto-optimal fronts obtained by MGSO-ACL in different scenarios given by UT, LHS and SR method are shown in Fig. 2. It is clear that our UT method approximates the LHS to a great extent, while the means and STDs of fuel cost obtained by SR method can

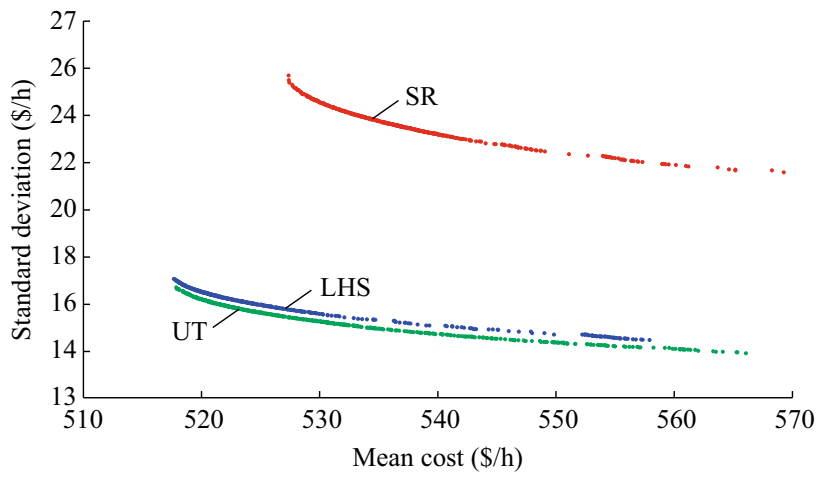

Fig. 2 Pareto-optimal fronts obtained by MGSO-ACL with different scenarios given by UT, LHS and SR method for Case 1 
not reflect the actual situation since SR distorts probability distributions of the random input variables. Moreover, as the sample number of the UT method is only $4.3 \%$ that of LHS and the execution time for one sample is the same, the execution time of the UT method is also only $4.3 \%$ of LHS, which means UT is very efficient in tackling the uncertainties.

Figure 3 shows the Pareto-optimal fronts obtained by MGSO-ACL and NSGA-II, respectively. For the metric comparisons between the two algorithms, the index of hypervolume (HV), the mean Euclidian distance (MED), the spacing index (SI), the number of Pareto-optimal solutions (NPS) are addressed to evaluate the quality of their Pareto-optimal fronts. The reference points for HV and MED are set as $(576,16.8)$ and $(517,14.0)$, respectively. According to the metric comparison results shown in Table 2, MGSO-ACL can find more Pareto-optimal solutions than NSGA-II under the same number of power flow calculations. Meanwhile, the values of HV and MED show that the Pareto-optimal front obtained by MGSOACL has better quality of convergence and diversity than that obtained by NSGA-II. In addition, SI regarding to MGSO-ACL is smaller, which means the Pareto-optimal solutions obtained by MGSO-ACL are distributed more evenly. Therefore, it is demonstrated that MGSO-ACL can find a better set of Pareto-optimal solutions, compared with NSGA-II. Moreover, the constraint violation objective values are 0 for all Pareto-optimal solutions, verifying the effectiveness of our constraint handling method.

As for the Pareto-optimal solutions obtained by MGSOACL, the decision making method, IEW, is applied to

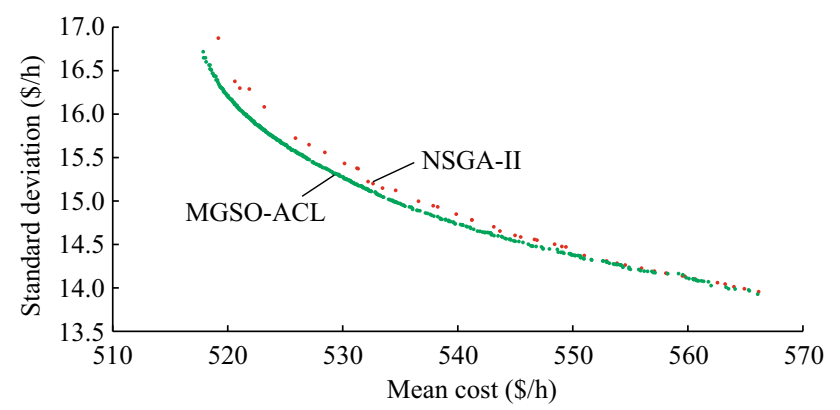

Fig. 3 Pareto-optimal fronts obtained by MGSO-ACL and NSGA-II with UT method for Case 1

Table 2 Metrics comparisons of Pareto-optimal fronts obtained by MGSO-ACL and NSGA-II

\begin{tabular}{llllr}
\hline Algorithm & HV & MED & Spacing & NPS \\
\hline MGSO-ACL & $\mathbf{9 7 . 8}$ & $\mathbf{1 5 . 9}$ & $\mathbf{1 1 . 9}$ & $\mathbf{4 3 7}$ \\
NSGA-II & 90.3 & 28.8 & 16.2 & 50 \\
\hline
\end{tabular}

Note: The bold fonts of values indicate the better results determine the final dispatch solution. After calculating the aggregation assessment value of each Pareto solution according to the computing procedure in Section 3.2, the solution with objective values $(534.0,15.0)$ is determined, and the errorbars of voltage magnitudes at all buses corresponding to this solution are shown in Fig. 4, where the STDs are multiplied by 10 for better observation. According to the obtained results and referring to the network topology, it is obvious that the integration of wind and solar farms in some buses results in uncertainty in all buses. In addition, the STDs of voltage magnitudes in buses which have wind or solar farms and in buses near to them topologically are higher than others.

\subsection{Case 2: Correlated input variables}

In this case the correlation between wind and solar power are taken into consideration to investigate what effects can be imposed to system by their negative correlation. Based on the statistical results in [35], the correlation coefficient of wind and solar power is set to be -0.2 .

The Pareto-optimal fronts obtained by MGSO-ACL with different scenarios given by UT, LHS and SR method are shown in Fig. 5. It is clear that the means and STDs of fuel cost obtained by the UT method coincides those of LHS. However, the Pareto-optimal front given by SR method are significantly different with LHS, indicating the poor ability of SR to handle the case with correlated input variables.

Comparing Fig. 5 with Fig. 2, it reveals that the negative correlation relationship of wind and solar power reduces the STD of fuel cost while has little influence on the mean value of fuel cost. As for the Pareto-optimal solutions obtained by MGSO-ACL in this case, the IEW is also applied to determine the final dispatch solution (533.4, 13.3). In order to further illustrate the effects imposed to regional IES by the negative correlation of wind and solar power, the final solutions obtained in the two cases are substituted into their sample points respectively, and the fuel cost in each sample are calculated using the nonlinear transformation process in Fig. 1. Figure 6 gives the fuel

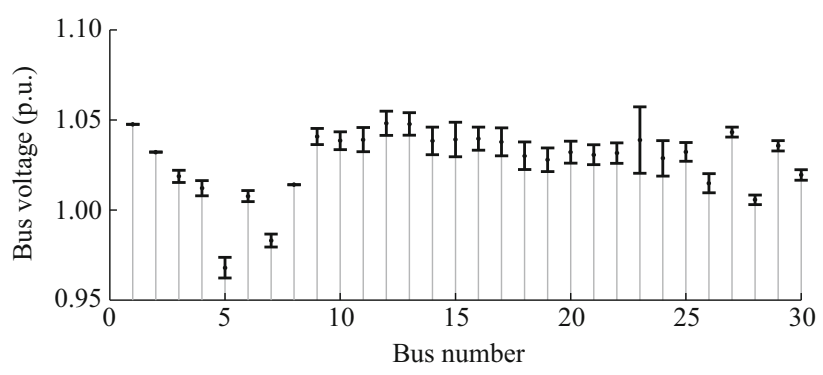

Fig. 4 Errorbars of voltage magnitudes at all buses for Case 1 


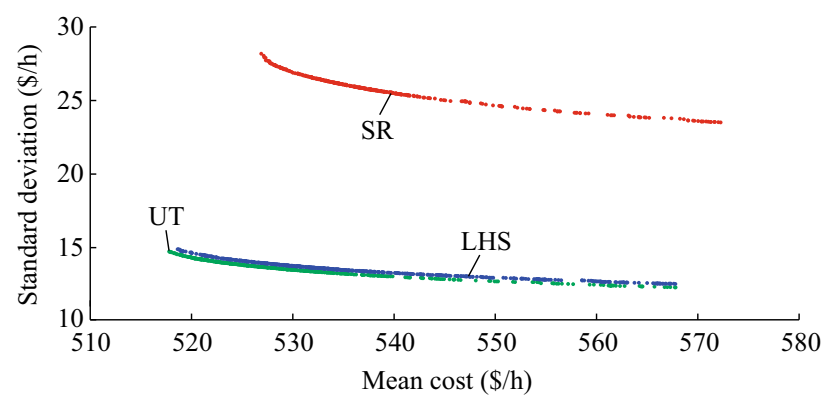

Fig. 5 Pareto-optimal fronts obtained by MGSO-ACL with different scenarios given by UT, LHS and SR method for Case 2

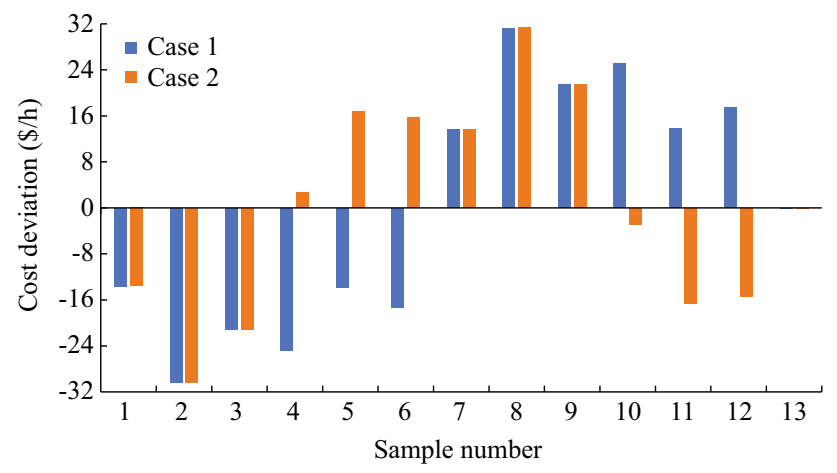

Fig. 6 Fuel cost deviations from the mean cost in 13 sample points corresponding to the final dispatch solutions for Case 1 and Case 2

cost deviations from the mean cost in 13 sample points for each case.

An additional advantage of the UT method is that it is convenient to investigate how the uncertainty of input variables caused the output variables to be uncertain. Therefore, we substitute the final solutions of both cases into their own sample points, and calculate the emissions of $\mathrm{CO}_{2}, \mathrm{NO}_{x}$ and $\mathrm{SO}_{2}$ in each sample by replacing the fuel cost function in the nonlinear transformation process of Fig. 1 with the following emission functions, respectively.

$$
\begin{aligned}
E_{\mathrm{CO}_{2}} & =\sum_{i=1}^{N_{\mathrm{G}}}\left(\tau_{1 i}+\tau_{2 i} P_{\mathrm{G}_{i}}+\tau_{3 i} P_{\mathrm{G}_{i}}^{2}\right) \\
E_{\mathrm{NO}_{x}} & =\sum_{i=1}^{N_{\mathrm{G}}} 10^{-2}\left(\tau_{4 i}+\tau_{5 i} P_{\mathrm{G}_{i}}+\tau_{6_{i}} P_{\mathrm{G}_{i}}^{2}+\epsilon_{i} e^{\xi_{i} P_{\mathrm{G}_{i}}}\right) \\
E_{\mathrm{SO}_{2}} & =\sum_{i=1}^{N_{\mathrm{G}}} 10^{-2}\left(\tau_{7 i}+\tau_{8 i} P_{\mathrm{G}_{i}}+\tau_{9 i} P_{\mathrm{G}_{i}}^{2}\right)
\end{aligned}
$$

where $\tau_{1 i}, \tau_{2 i}, \ldots, \tau_{9 i}$ are the cost coefficients for the emissions of $\mathrm{CO}_{2}, \mathrm{NO}_{x}$ and $\mathrm{SO}_{2}$, respectively.

The emissions of $\mathrm{CO}_{2}, \mathrm{NO}_{x}$ and $\mathrm{SO}_{2}$ in 13 samples for Case 1 and Case 2 are plotted in Fig. 7. It can be observed that the emissions of $\mathrm{CO}_{2}$ and $\mathrm{SO}_{2}$ are almost invariable in the different scenarios, indicating that the uncertainty of

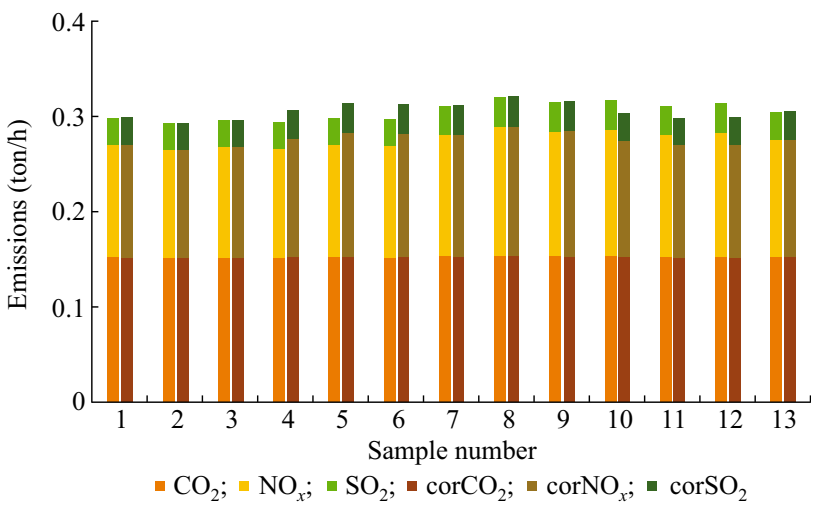

Fig. 7 Emissions of $\mathrm{CO}_{2}, \mathrm{NO}_{x}$ and $\mathrm{SO}_{2}$ in 13 sample points

\begin{tabular}{|c|c|c|c|c|c|}
\hline \multirow[t]{2}{*}{ Objective } & \multicolumn{2}{|l|}{ Case 1} & \multicolumn{2}{|l|}{ Case 2} & \multirow[t]{2}{*}{ Original } \\
\hline & Mean & STD & Mean & STD & \\
\hline$F$ & 534.0 & 15.0 & 533.4 & 13.3 & 802.3 \\
\hline$E_{\mathrm{CO}_{2}}$ & 0.1527 & 0.0006 & 0.1525 & 0.0005 & 0.2842 \\
\hline$E_{\mathrm{NO}_{x}}$ & 0.1236 & 0.0053 & 0.1240 & 0.0047 & 0.3623 \\
\hline$E_{\mathrm{SO}_{2}}$ & 0.0292 & 0.0010 & 0.0296 & 0.0009 & 0.0859 \\
\hline VD & 0.8297 & 0.0132 & 0.8025 & 0.0103 & 0.7926 \\
\hline$L_{\text {index }}$ & 0.1543 & 0.0018 & 0.1557 & 0.0014 & 0.1387 \\
\hline
\end{tabular}
corresponding to the final dispatch solutions for Case 1 and Case 2

Table 3 Means and STDs obtained in Case 1 and Case 2

wind and solar power has little impact on them. In the meanwhile, the emission of $\mathrm{NO}_{x}$ changes in different scenarios. The reason behind this phenomenon lies in that the emission function of $\mathrm{NO}_{x}$ is the summation of a quadratic function and an exponential function, while the exponential function is more sensitive to the fluctuation of power outputs.

To investigate the impact of the integration of REs on power system risk indices, the means and STDs of voltage deviations (VD) and voltage stability index $\left(L_{\text {index }}\right)$ corresponding to the final dispatch solutions of Case 1 and Case 2 are also calculated. The obtained values are listed in Table 3, and the original values for these objectives are also given for comparison. Here, the original values are obtained by minimizing the fuel cost in the original regional IES and the best individual is used to calculate the other objective values. From Table 3, it can be seen that integrating REs can effectively improve the economic and environmental benefits, but it also increases the risk of IES. Moreover, the uncertainty caused by the REs is also the risk that should be assessed. In addition, the STDs of the objective values of Case 2 are all smaller than those of Case 1, which again verifies that the complementary nature of wind and solar power can reduce the uncertainty of the overall system. 


\section{Conclusion}

This paper has provided a UT-MS model for the IES optimal operation problem considering correlative wind and solar power. The MGSO-ACL algorithm is applied along with a constraint handling method to deal with the UT-MS model. By comparing all the Pareto-optimal solutions using the decision making method, IEW, a final dispatch solution is then determined. The following conclusions have been drawn via the simulation studies conducted on a test IES.

First, the UT is more efficient than the LHS method since it uses much less sample points, and more accurate than the SR method in calculating the statistical properties of output variables, while having the ability to handle correlated input variables.

Secondly, the MGSO-ACL-CHT algorithm can obtain superior Pareto-optimal solutions in terms of convergence and diversity, and the constraint handling technique can ensure the Pareto-optimal solutions satisfy all the constraints for all scenarios.

Thirdly, the impact of correlation among the random input variables on systems has also been investigated in this paper, and the results have shown that the negative correlated relationship between wind and solar power reduces the STDs of system variables but has little influence on the mean values of system variables.

Furthermore, we have also compared the fuel costs, emissions of $\mathrm{CO}_{2}, \mathrm{NO}_{x}$ and $\mathrm{SO}_{2}$, voltage deviations and voltage stability indices corresponding to the dispatch solutions of the modified and original regional IES, and it reveals that integrating REs can effectively improve the economic and environmental benefits, but also increase the risk for the IES optimal operation problem.

In our future work, we will apply the proposed UT-MS model to tackle the problem of $N-k$ security constrained unit commitment considering renewable energy resources penetrating into IES, so as to increase the proportion of renewable energy consumption while guaranteeing the economy and stability of the IES operation.

Acknowledgements This project was supported by the State Key Program of National Natural Science Foundation of China (No. 51437006), the Fundamental Research Funds for the Central Universities and the China Postdoctoral Science Foundation (No. 2017M622690).

Open Access This article is distributed under the terms of the Creative Commons Attribution 4.0 International License (http:// creativecommons.org/licenses/by/4.0/), which permits unrestricted use, distribution, and reproduction in any medium, provided you give appropriate credit to the original author(s) and the source, provide a link to the Creative Commons license, and indicate if changes were made.

\section{References}

[1] Bahmani-Firouzi B, Farjah E, Azizipanah-Abarghooee R (2013) An efficient scenario-based and fuzzy self-adaptive learning particle swarm optimization approach for dynamic economic emission dispatch considering load and wind power uncertainties. Energy 50:232-244

[2] Zhang X, Shahidehpour M, Alabdulwahab A et al (2016) Hourly electricity demand response in the stochastic day-ahead scheduling of coordinated electricity and natural gas networks. IEEE Trans Power Syst 31(1):592-601

[3] Reddy SS, Bijwe P, Abhyankar A (2015) Real-time economic dispatch considering renewable power generation variability and uncertainty over scheduling period. IEEE Syst J 9(4):1440-1451

[4] Wu L, Shahidehpour M, Li Z (2012) Comparison of scenariobased and interval optimization approaches to stochastic SCUC. IEEE Trans Power Syst 27(2):913-921

[5] Reddy SS, Sandeep V, Jung CM (2017) Review of stochastic optimization methods for smart grid. Front Energy 11(2):197-209

[6] Miranda V, Hang PS (2005) Economic dispatch model with fuzzy wind constraints and attitudes of dispatchers. IEEE Trans Power Syst 20(4):2143-2145

[7] Liang RH, Liao JH (2007) A fuzzy-optimization approach for generation scheduling with wind and solar energy systems. IEEE Trans Power Syst 22(4):1665-1674

[8] Liang RH, Tsai SR, Chen YT et al (2011) Optimal power flow by a fuzzy based hybrid particle swarm optimization approach. Electr Power Syst Res 81(7):1466-1474

[9] Momoh A, Reddy SS (2014) Review of optimization techniques for renewable energy resources. In: Proceedings of 2014 IEEE symposium on power electronics and machines for wind and water applications, Milwaukee, USA, 24-26 July 2014, pp 1-8

[10] $\mathrm{Li} \mathrm{YZ,} \mathrm{Wu} \mathrm{QH,} \mathrm{Jiang} \mathrm{L} \mathrm{et} \mathrm{al} \mathrm{(2016)} \mathrm{Optimal} \mathrm{power} \mathrm{system}$ dispatch with wind power integrated using nonlinear interval optimization and evidential reasoning approach. IEEE Trans Power Syst 31(3):2246-2254

[11] Reddy SS, Park JY, Jung CM (2016) Optimal operation of microgrid using hybrid differential evolution and harmony search algorithm. Front Energy 10(3):355-362

[12] Fu YM, Liu MB, Li LC (2016) Multi-objective stochastic economic dispatch with variable wind generation using scenario-based decomposition and asynchronous block iteration. IEEE Trans Sustain Energy 7(1):139-149

[13] Reddy SS, Momoh A (2015) Realistic and transparent optimum scheduling strategy for hybrid power system. IEEE Trans Smart Grid 6(6):3114-3125

[14] Aghaei J, Niknam T, Azizipanah-Abarghooee R et al (2013) Scenario-based dynamic economic emission dispatch considering load and wind power uncertainties. Int $\mathbf{J}$ Electr Power Energy Syst 47:351-367

[15] Li YZ, Li MS, Wu QH (2014) Optimal reactive power dispatch with wind power integrated using group search optimizer with intraspecific competition and lévy walk. J Mod Power Syst Clean Energy 2(4):308-318

[16] Li J, Ye L, Zeng Y et al (2016) A scenario-based robust transmission network expansion planning method for consideration of wind power uncertainties. CSEE J Power Energy Syst 2(1):11-18

[17] Lubin M, Dvorkin Y, Backhaus S (2015) A robust approach to chance constrained optimal power flow with renewable generation. IEEE Trans Power Syst 31(5):3840-3849 
[18] Bai XQ, Qu TY, Qiao W (2015) Robust AC optimal power flow for power networks with wind power generation. IEEE Trans Power Syst 31(5):4163-4164

[19] Xiang Y, Liu YB, Liu JY et al (2016) A chance-constrained optimization model for determining renewables penetration limit in power systems. Electr Power Compos Syst 44(7):701-712

[20] Zhang H, Li P (2011) Chance constrained programming for optimal power flow under uncertainty. IEEE Trans Power Syst 26(4):2417-2424

[21] Zhang N, Kang CQ, Xia Q et al (2015) A convex model of riskbased unit commitment for day-ahead market clearing considering wind power uncertainty. IEEE Trans Power Syst 30(3): 1582-1592

[22] Wang Q, Yang M, Wen FH et al (2013) Risk-based securityconstrained economic dispatch in power systems. J Mod Power Syst Clean Energy 1(2):142-149

[23] Shaha R, Kothari DP, Chandrakar VS (2016) Optimization of renewable energy sources for hybrid power generation. In: Proceedings of 2016 biennial international conference on power and energy systems: towards sustainable energy (PESTSE), Bangalore, India, 21-23 January 2016, pp 1-5

[24] Alabdulwahab A, Abusorrah A, Zhang X et al (2015) Coordination of interdependent natural gas and electricity infrastructures for firming the variability of wind energy in stochastic dayahead scheduling. IEEE Trans Power Syst 6(2):606-615

[25] Reddy SS, Bijwe PR (2016) Day-ahead and real time optimal power flow considering renewable energy resources. Int J Electr Power Energy Syst 82:400-408

[26] Growe-Kuska N, Heitsch H, Romisch W (2003) Scenario reduction and scenario tree construction for power management problems. In: Proceedings of 2003 IEEE Bologna power tech conference, Bologna, Italy, 23-26 June 2003, pp 152-158

[27] Julier SJ, Uhlmann JK (2004) Unscented filtering and nonlinear estimation. Proc IEEE 92(3):401-422

[28] Aien M, Rashidinejad M, Firuz-Abad MF (2015) Probabilistic optimal power flow in correlated hybrid wind-power systems: a review and a new approach. Renew Sustain Energy Rev 41:1437-1446

[29] Li YZ, Wu QH, Li MS et al (2014) Mean-variance model for power system economic dispatch with wind power integrated. Energy 72:510-520

[30] Zheng JH, Chen JJ, Wu QH et al (2015) Multi-objective optimization and decision making for power dispatch of a largescale integrated energy system with distributed DHCS embedded. Appl Energy 154:369-379

[31] Lange M (2005) On the uncertainty of wind power predictionsanalysis of the forecast accuracy and statistical distribution of errors. J Sol Energy Eng 127(2):177-184

[32] Bludszuweit H, Domnguez-Navarro JA, Llombart A (2008) Statistical analysis of wind power forecast error. IEEE Trans Power Syst 23(3):983-991

[33] Ziadi Z, Oshiro M, Senjyu T et al (2014) Optimal voltage control using inverters interfaced with $\mathrm{PV}$ systems considering forecast error in a distribution system. IEEE Trans Sustain Energy 5(2):682-690

[34] Li MS, Wu QH, Ji TY et al (2014) Stochastic multi-objective optimization for economic-emission dispatch with uncertain wind power and distributed loads. Electr Power Syst Res 116:367-373

[35] Widén J (2011) Correlations between large-scale solar and wind power in a future scenario for Sweden. IEEE Trans Sustain Energy 2(2):177-184
[36] Deb K (2002) Salient issues of multi-objective evolutionary algorithms. Multi Object Optim Evolut Algorithms 8:347-348

[37] Basu M (2008) Dynamic economic emission dispatch using nondominated sorting genetic algorithm-II. Int J Electr Power Energy Syst 30(2):140-149

[38] Niknam T, Narimani M, Aghaei J et al (2012) Improved particle swarm optimisation for multi-objective optimal power flow considering the cost, loss, emission and voltage stability index. IET Gen Transm Distrib 6(6):515-527

[39] Ben Said L, Bechikh S, Ghedira K (2010) The r-dominance: a new dominance relation for interactive evolutionary multicriteria decision making. IEEE Trans Evolut Comput 14(5):801-818

[40] Roy R, Jadhav H (2015) Optimal power flow solution of power system incorporating stochastic wind power using Gbest guided artificial bee colony algorithm. Int J Electr Power Energy Syst 64:562-578

[41] Yuan XH, Tian H, Yuan YB et al (2015) An extended NSGA-III for solution multi-objective hydro-thermal-wind scheduling considering wind power cost. Energy Convers Manag 96:568-578

[42] Shannon CE (2001) A mathematical theory of communication. ACM SIGMOBILE Mobile Comput Commun Rev 5(1):3-55

[43] Yu H, Chung CY, Wong KP et al (2009) Probabilistic load flow evaluation with hybrid latin hypercube samplingand cholesky decomposition. IEEE Trans Power Syst 24(2):661-667

Jiehui ZHENG obtained his B.E. degree in Electrical Engineering from Huazhong University of Science and Technology, Wuhan, China, in 2012, and his Ph.D. degree at the same area in South China University of Technology (SCUT), Guangzhou, China in 2017. He is currently a research assistant in SCUT. His research interests include optimization algorithms, decision making methods and their applications on integrated energy systems.

Yanni KOU received the B.E. degree in Electrical Engineering from Huazhong University of Science and Technology, Wuhan, China, in 2014. She obtained the M.S. degree at the same area in South China University of Technology, Guangzhou, China. Her research interests include stochastic optimization, multi-objective optimization and its application on integrated energy systems.

Mengshi LI received the M.Sc.(Eng.) (with distinction) and Ph.D. degrees in electrical engineering and electronics from the University of Liverpool, Liverpool, U.K., in 2005 and 2010, respectively. He is currently an associate professor with the School of Electric Power Engineering, South China University of Technology, Guangzhou, China. His research interests include computational intelligence and their applications in power systems.

Qinghua WU obtained an M.Sc.(Eng) degree in Electrical Engineering from Huazhong University of Science and Technology, Wuhan, China, in 1981. From 1981 to 1984, he was appointed Lecturer in Electrical Engineering in the University. He obtained a Ph.D. degree in Electrical Engineering from The Queen's University of Belfast (QUB), Belfast, U.K. in 1987. He worked as a Research Fellow and subsequently a Senior Research Fellow in QUB from 1987 to 1991. He joined the Department of Mathematical Sciences, Loughborough University, Loughborough, U.K. in 1991, as a Lecturer, subsequently he was appointed Senior Lecturer. In September, 1995, he joined The University of Liverpool, Liverpool, U.K. to take up his appointment to the Chair of Electrical Engineering in the Department of Electrical 
Engineering and Electronics. Now he is with the School of Electric Power Engineering, South China University of Technology, Guangzhou, China, as a Distinguished Professor and the Director of Energy Research Institute of the University. Professor Wu has authored and coauthored more than 440 technical publications, including 220 journal papers, 20 book chapters and 3 research monographs published by Springer. He is a Fellow of IEEE, Fellow of IET, Chartered Engineer and Fellow of InstMC. His research interests include nonlinear adaptive control, mathematical morphology, evolutionary computation, power quality and power system control and operation. 